\title{
Enhanced optical trapping
}

Butaite, Une, Gibson, Graham, Ho, Ying-Lung, Taverne, Mike, Taylor, Jonathan, et al.

Une G. Butaite, Graham M. Gibson, Ying-Lung D. Ho, Mike Taverne, Jonathan M. Taylor, David B. Phillips, "Enhanced optical trapping," Proc. SPIE 11522, Optical Manipulation and Structured Materials Conference 2020, 115221I (15 June 2020); doi: 10.1117/12.2573773

SDIE Event: SPIE Technologies and Applications of Structured Light, 2020, Yokohama, Japan 


\title{
Enhanced optical trapping
}

\author{
Une G. Butaite*a, Graham M. Gibson ${ }^{\mathrm{a}}$, Ying-Lung D. Ho ${ }^{\mathrm{b}}$, Mike Taverne ${ }^{\mathrm{b}}$, Jonathan M. Taylor ${ }^{\mathrm{a}}$, \\ David B. Phillips ${ }^{\mathrm{c}}$

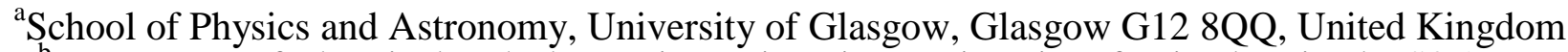 \\ ${ }^{\mathrm{b}}$ Department of Electrical and Electronic Engineering, University of Bristol, Bristol BS8 1UB, \\ United Kingdom \\ ${ }^{\mathrm{c}}$ School of Physics and Astronomy, University of Exeter, Exeter EX4 4QL, United Kingdom
}

\begin{abstract}
Optical tweezers have contributed substantially to the advancement of micro-manipulation. However, they do have restrictions, mainly the limited range of materials that yield to optical trapping. Here we propose a method of employing optically trapped objects to manipulate the surrounding fluid and thus particles freely diffusing within it. We create and investigate a reconfigurable active-feedback system of optically trapped actuators, capable of manipulating translational and rotational motion of one or more nearby free objects.
\end{abstract}

Keywords: holographic optical tweezers, hydrodynamic manipulation, micro-manipulation

*email: u.butaite.1@ research.gla.ac.uk

\section{Introduction}

Optical tweezers have been proven time and again to be extremely useful tools for micro-manipulation [1] [2] [3]. However, they are limited to certain materials that can be optically trapped and are inevitably harmful to sensitive biological specimens.

We show here that these limitations can be overcome by employing optically trapped actuators to take control of the surrounding fluid and any freely-floating objects within it.

\section{Concept and Theory}

\section{Micro-rotors and system setup}

We designed micro-rotors, as seen in Figure 1, in a wheel-like shape with three spokes. Incorporated on each spoke is a prolate spheroidal handle allowing optical trapping. By directing an optical beam through each of the handles we have complete control of the translational and rotational motion or the rotor, and, by spinning it, can actuate the surrounding fluid.

The micro rotors were fabricated using direct laser writing [4]. This technology is not, however, readily available, so we also demonstrate that micro-rotors can be replaced with three-bead constellation rotors, as can be seen in Figure 1(c), where each bead is optically trapped and constrained to move in a circle. (a)
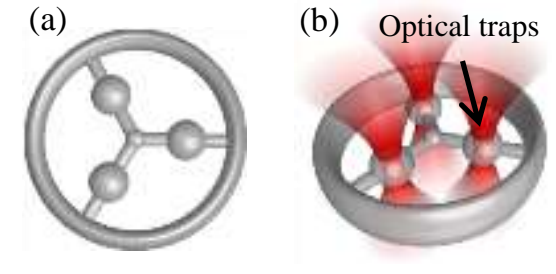

(c)

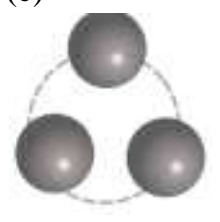

Figure 1. (a) Schematic of a micro-rotor with three spokes. The rotor can be optically trapped and manipulated by passing three focused laser beams through each of the handle spheroids as seen in (b). (c) A three-bead constellation rotor, which can replace the laser printed micro-rotors.

Knowledge of hydrodynamic coupling between colloidal particles allows us to spin the rotors in such a way that the motion of the target object can be controlled as we desire. As illustrated in Figure 2, for 2-dimensional translational control we use two micro-rotors, positioned so that they individually address the $\mathrm{x}$ and $\mathrm{y}$ degrees of freedom of the target. By combining the effects of the two spinning rotors we have full control of the target in two dimensions. 

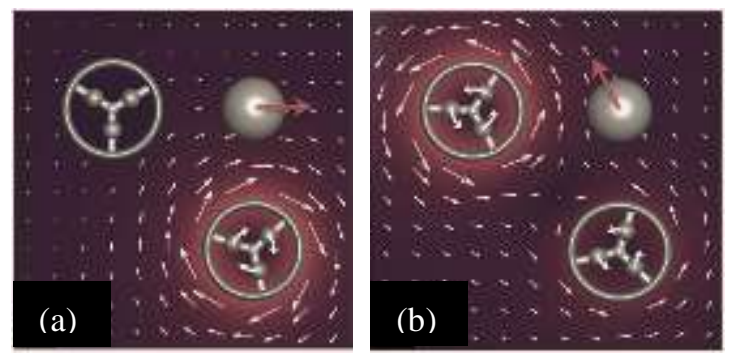

Figure 2. 2-dimensional hydrodynamic control. (a) Spinning the bottom rotor will move the target along a straight line (provided the target bead is directly above it). (b) Combining the rotation of the two rotors allows us to move the target in any desired direction.

\section{Hydrodynamic feedback loop}

Our hydrodynamic manipulation feedback loop consists of 4 steps. First, the position of the target is registered using live video tracking and the distance from target's desired location is noted. Then, rotation rates of micro-rotors that will push the target towards the desired location are calculated. The rotors will be moved by updating the locations of the optical traps. For this, the hologram displayed on the spatial light modulator (SLM) has to be updated. Once the rotors are in motion, they affect the target, its position is registered again and the feedback loop is repeated.

To execute the second step we need to model hydrodynamic interactions between the micro-rotors and the target. We do this by mathematically treating each rotor as a single spherical particle and using a model that describes the hydrodynamic coupling between an arbitrary number of spheres [5]. It can be shown [6] that the velocity of the target $\boldsymbol{v}_{t}$ is given by:

$\boldsymbol{v}_{t}=\boldsymbol{C}_{t r} \boldsymbol{\omega}_{r}$,

where $\boldsymbol{\omega}_{r}$ is a column vector representing the rotation rates of the rotors and $\boldsymbol{C}_{t r}$ is a matrix containing all the information about hydrodynamic coupling in the system. The components of $\boldsymbol{C}_{t r}$ are dependent on the relative locations between the interacting objects and therefore have to be recalculated in each iteration. To determine how the micro-rotors need to be spun, Eqn. (1) is solved for $\boldsymbol{\omega}_{r}$. This equation can of course be extended to include any number of targets and rotors.
Note, that while, in principle, the system can be extended to address the z-dimension of the target, we eliminate this need by performing experiments on target particles which are sedimented to the bottom of the sample cell.

\section{Results and Discussion}

To test our method, we first attempted to suppress the Brownian fluctuations of a single $5 \mu \mathrm{m}$ radius silica bead, by confining it to a single spot on the bottom of the sample cell. We do this successfully with the resulting bead motion limited to a standard deviation of $79 \mathrm{~nm}$ as shown in Figure 3. Naturally, the target can also be moved along any trajectory, so long as it is sufficiently close to the rotors.

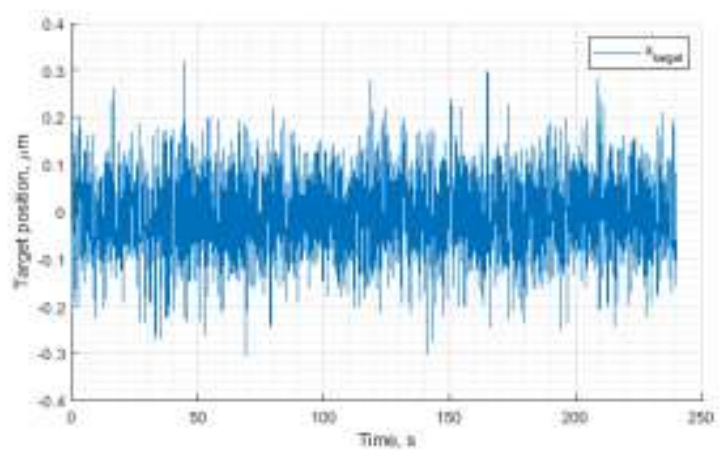

Figure 3. $\mathrm{x}$-coordinate trace of a $5 \mu \mathrm{m}$ radius target bead being hydrodynamically held at a single location for a few minutes. The standard deviation of this motion is $79 \mathrm{~nm}$.

We further show in Figure 4 that the same experiments can be performed on two target particles. Particularly exciting is the manipulation of two silica beads, performing a range of different motions: moving both beads anti-clockwise, pushing and pulling them apart, following concentric trajectories in opposite directions, moving the centre of mass with fixed separation between the beads and holding one bead stationary while the other one is moving. 

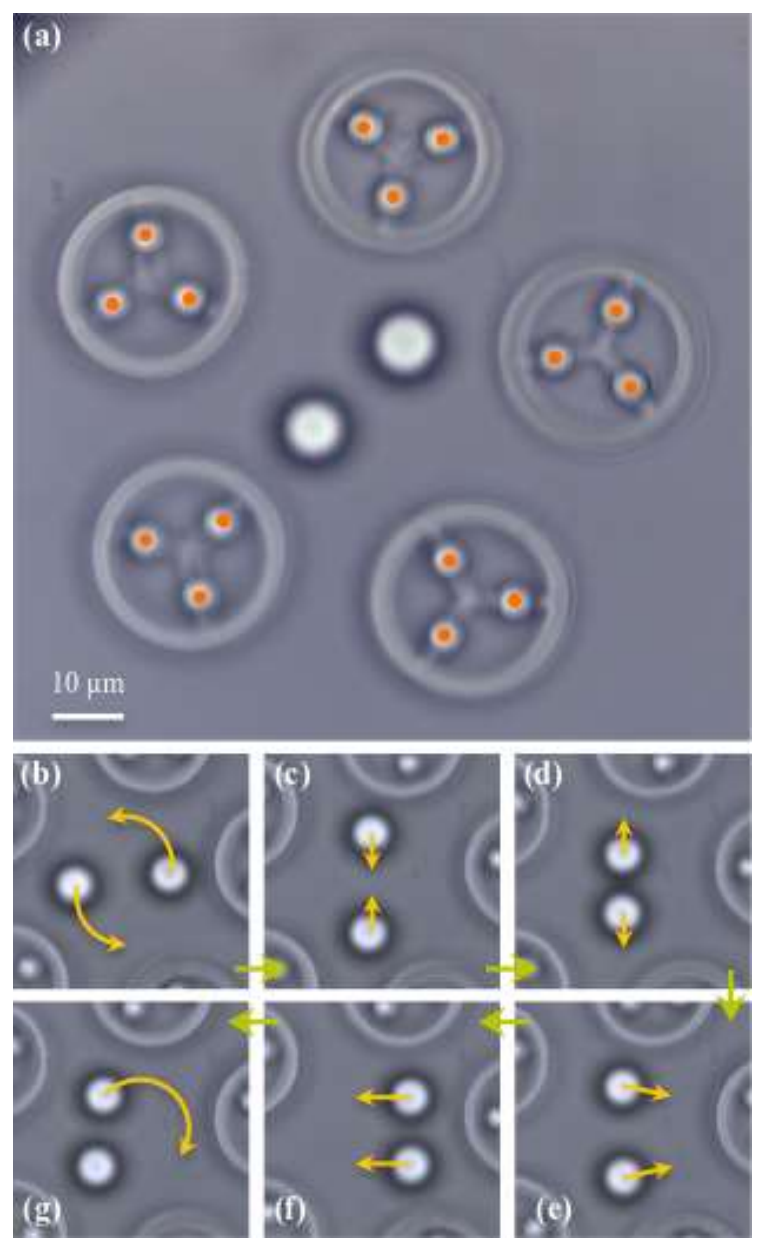

Figure 4. Hydrodynamic manipulation of two $5 \mu \mathrm{m}$ radius target beads using five micro-rotors. (a) The setup of manipulation, with orange dots indicating locations of optical traps. (b-g) Motions of the two beads that can be generated using the five microrotors.

Further results, including performance of bead constellation rotors, long-range translation of a target across the sample and orientation control of a yeast cell, with accompanying videos can be found in [6].

\section{Conclusion}

We have designed and demonstrated a system that extends the capabilities of holographic optical tweezers, by facilitating them for hydrodynamic manipulation. Our method allows translational and rotational manipulation of one or more freely diffusing target particles, irrespective of their material, at the same time offering a minimally invasive technique for controlling biological specimens, without exposing them to intense laser light.

\section{References}

[1] U. Bockelmann, P. Thomen, B. Essevaz-Roulet, V. Viasnoff and F. Heslot, "Unzipping DNA with optical tweezers: high sequence sensitivity and force flips," Biophysical journal, vol. 82, pp. 1537-1553, 2002.

[2] C. O. Mejean, A. W. Schaefer, E. A. Millman, P. Forscher and E. R. Dufresne, "Multiplexed force measurements on live cells with holographic optical tweezers," Optics express, vol. 17, pp. 6209-6217, 2009.

[3] M. Simons, M. R. Pollard, C. D. Hughes, A. D. Ward, B. Van Houten, M. Towrie, S. W. Botchway, A. W. Parker and N. M. Kad, "Directly interrogating single quantum dot labelled UvrA 2 molecules on DNA tightropes using an optically trapped nanoprobe," Scientific reports, vol. 5, p. 18486, 2015.

[4] B. H. Cumpston, S. P. Ananthavel, S. Barlow, D. L. Dyer, J. E. Ehrlich, L. L. Erskine, A. A. Heikal, S. M. Kuebler, I.-Y. S. Lee, D. McCord-Maughon and others, "Two-photon polymerization initiators for three-dimensional optical data storage and microfabrication," Nature, vol. 398, pp. 51-54, 1999.

[5] P. Mazur and W. Van Saarloos, "Many-sphere hydrodynamic interactions and mobilities in a suspension," Physica A: Statistical Mechanics and its Applications, vol. 115, pp. 21-57, 1982.

[6] U. G. Būtaite, D. B. Phillips, J. M. Taylor, G. M. Gibson, Y.-L. D. Ho and M. Taverne, "Indirect optical trapping using light driven micro-rotors for reconfigurable hydrodynamic manipulation," Nature Communications, vol. 10, no. 1, p. 1215, 2019. 